\title{
Comparison of the effect of dexmedmotidine and ketamine on controlling pain after cesarean section via intra-peritoneal method
}

\author{
Alireza Kamali, ${ }^{1}$ Maryam Maktabi, ${ }^{2}$ Zoha Khademi, ${ }^{1}$ and Taha Fereidooni ${ }^{1}$
}

\author{
'Department of Anesthesiology and Critical Care, Arak University of Medical Sciences, Arak, Iran. \\ 2Department of Gynecology, Arak University of Medical Sciences, Arak, Iran. \\ Correspondence to Maryam Maktabi (email: dralirezakamalianesthesiology@gmail.com). \\ (Submitted: 26 April 2019 - Revised version received: 12 May 2019 - Accepted: 23 June 2019 - Published online: 26 August 2019)
}

\begin{abstract}
objective The present study aimed to compare the effect of dexmedmotidine and ketamine on controlling pain after cesarean section via intra-peritoneal method.

Methods In this clinical and double-blind clinical trial, patients were randomly divided into two groups (dexmedmotidine and ketamine). In the first group, $5 \mathrm{mg} / \mathrm{kg}$ ketamine and $1 \mathrm{mg} / \mathrm{kg}$ dexmedetomidine were injected in $100 \mathrm{mg}$ normal saline. Pain score was measured on the basis of the visual analog pain scale during the recovery at 4,6 and $12 \mathrm{~h}$ after the surgery. The data were then analyzed by SPSS (version 20).

Results A total of 70 patients participated in the study. The results showed that the mean pain scores were the same in different postoperative hours in patients $(P \geq 0.05)$. The mean opioid use in the ketamine group was lower than inter-peritoneal dexmedmotidine $(P=0.03)$. Moreover, the mean postoperative analgesia in the ketamine group was higher than inter-peritoneal dexmedmotidine $(P=0.04)$.

Conclusion According to the results, the mean opioid consumed in the ketamine group was less than inter-peritoneal dexmedmotidine. Additionally, the mean postoperative analgesia in the ketamine group was higher than that of inter-peritoneal dimethomidine. Therefore, it can be concluded that ketamine has a better effect on reducing pain after cesarean section.

Keywords intra-peritoneal, dexmedmotidine, cesarean section, ketamine, pain control
\end{abstract}

\section{Introduction}

Pain is a completely mental experience, which produces adverse hemodynamic and metabolic responses in patients. ${ }^{1,2}$ Millions of people around the world undergo surgery and then experience post-surgical pain. Postoperative pain leads to harmful effects, such as atleticas, thrombosis, ischemic myocardium, cardiac arrhythmias, water and electrolyte disorders, urinary retention and ileus. ${ }^{3,4}$ One of the most commonly used surgical procedures is cesarean section. Caesarean section is used in cases where delivery is impossible or if there is a risk to the mother and the baby. This procedure has played an important role in reducing maternal and fetal deaths and complications over the last century. ${ }^{5,6}$ Cesarean section is performed in two ways, including general anesthesia or regional anesthesia.? One of the problems of cesarean section is acute postoperative pain that causes unpleasant psychological responses such as anxiety, sadness, aggression, insomnia, and lack of logical connection with the physician and nurse. It may also reduce breastfeeding and mother's tolerance for breastfeeding. ${ }^{8,9}$ One of the most important issues in cesarean section is to reduce postoperative pain. By reducing the pain, mothers will be able to perform their motherhood duties well, leading to earlier and more appropriate lactation. Opioid drugs such as morphine and pethidine, which are the most commonly used drugs to relieve postoperative pain, are associated with unpleasant complications such as addiction, respiratory arrest, resistance, nausea, vomiting, etc. Therefore, the use of alternative opioid drugs to prevent postoperative pain has always been pursued by surgeons and researchers. ${ }^{10}$ Genealogy is one of the widely used methods that reduces the effect of opioid drugs on the fetus. ${ }^{11}$ First identified in 1951 by Griffin, intra-perotone anesthesia is a new method for reducing postoperative pain. Various studies have focused on pain relief after laparoscopy, hysterectomy, and laparoscopic surgery via intra-peritoneal method. The systemic effect of this method begins two minutes after the intraperitoneal injection. ${ }^{12}$ Various adjuvants can be used, including dexmedmotidine and ketamine. Ketamine is an NMDA receptor antagonist and an anesthetic drug. ${ }^{13}$ Ketamine is also an intravenous anesthetic with an analgesic effect which stimulates the cardiovascular system with minimum respiratory suppression. ${ }^{14}$ Dexmedmotidine is analgesic, sedative, and antihypertensive. ${ }^{15}$ Dexmedmotidine and ketamine alone increase the duration of analgesia and reduce the use of narcotics. On the other hand, the intra-peritoneal method during childbirth has a significant effect on the health of the fetus because it reduces the likelihood of anesthetic drug transfer from mother to fetus. Given that all studies conducted on abdominal surgery have only investigated the effect of a single drug, we aimed to compare the effect of dexmedmotidine and ketamine on controlling pain after cesarean section via intra-peritoneal method.

\section{Materials and Methods}

This double-blind clinical trial was carried out on all patients who underwent cesarean section. All candidates for cesarean section referred to Taleghani Hospital in Arak, Iran. The candidates for cesarean section who entered the study were randomly divided into two groups (dexmedmotidine and ketamine).

\section{Inclusion Criteria}

1. Aged $18-35$ years.

2. ASA (American Society of Anesthesiologists).

3. Female candidates for cesarean section. 


\section{Exclusion Criteria}

1. Having a history of allergy to dexmedmotidine and ketamine.

2. Having heart and respiratory disease.

3 . Emergency cesarean section.

At first, all patients provided informed consent. Then, $5 \mathrm{mg} / \mathrm{kg}$ ketamine and $1 \mathrm{mg} / \mathrm{kg}$ dexmedmotidine were injected in the ketamine and dexmedmotidine group, respectively. In both groups, the normal saline solution mixed with drugs was injected into the patient's peritoneum. The pain was measured according to the Visual Analog Scale (VAS) at 4, 6 and $12 \mathrm{~h}$ after the operation. In this scale, zero expresses the lowest value and the 10 represents the highest value. It should be noted that the data were measured and recorded by a gynecologist who was unaware of the groupings. The drugs in each group were prepared by an anesthetist.

The sample size and number were calculated as follows:

$$
N=\frac{\left(Z_{1-\frac{\alpha}{2}}+Z_{1-\beta}\right)^{2}\left(\delta_{1}+\delta_{2}\right)^{2}}{\left(\mu_{1}+\mu_{2}\right)^{2}}=70
$$

Patients were divided into two groups $(n=35)$

\section{Data analysis}

The data were analyzed using SPSS (version 20), while descriptive statistics and $t$-test were used to analyze parametric and nonparametric data.

\section{Ethical considerations}

1. Obtaining a letter of introduction from the university's authorities to be introduced to the research centers.

2. Obtaining a letter of introduction from the authorities of the researcher centers.

3. The purpose of the study was described for all research units and written consent was obtained from them.

\section{Results}

A total of 70 patients were included in the study, who were classified into two groups. They were evaluated in terms of mean age. According to the results, the mean age in the dexmedmotidine group and ketamine group was $34.4 \pm 3.1$ and $35.1 \pm 4.7$ years, respectively $(P=0.6)$. There was no significant difference between the two groups in terms of mean age. It can be said that the mean age of patients was similar in the two groups.

Table 1 shows the comparison of pain scores between the two groups. Given the fact that $P<0.05$, there was no significant difference between the groups in scores of pain 4,6 , and $12 \mathrm{~h}$ after the recovery. The mean pain score was almost similar in patients at different hours after the surgery.

According to Table 2, there was a significant difference between the two groups in terms of drug abuse in $12 \mathrm{~h}$ after the surgery $(P=0.03)$, indicating that the mean drug in the ketamine group was lower than that of dexmedmotidine.

In Table 3, the mean duration of analgesia was evaluated and $P=0.04$ was significant in two both. This indicates that the

\begin{tabular}{lcccc}
\hline \multicolumn{2}{l}{ Table 1 Comparison of pain scores } & & \\
\cline { 1 - 3 } Group & Dexmedmotidine & Ketamine & \\
\cline { 1 - 2 } Pain & Mean \pm SD & Mean \pm SD & \\
\hline VAS & $0.0 \pm 0.0$ & $0.0 \pm 0.0$ & $\geq 0.05$ \\
VAS 4 h after the surgery & $1.1 \pm 0.65$ & $0.98 \pm 0.23$ & 0.2 \\
VAS 6 h after the surgery & $1.6 \pm 3.4$ & $1.7 \pm 3.1$ & 0.4 \\
VAS 12 h after the surgery & $1.1 \pm 3.7$ & $0.98 \pm 3.8$ & 0.6
\end{tabular}

\begin{tabular}{|c|c|c|c|}
\hline Group & Dexmedmotidine & Ketamine & \multirow{2}{*}{$P$} \\
\hline Variable & Mean \pm SD & Mean \pm SD & \\
\hline $\begin{array}{l}\text { Drug use } 12 \mathrm{~h} \text { after } \\
\text { the surgery }(\mathrm{mg})\end{array}$ & $35 \pm 4.4$ & $20.4 \pm 3$ & 0.03 \\
\hline
\end{tabular}

\begin{tabular}{lccc}
\hline \multicolumn{4}{l}{ Table 3. Comparison of the mean postoperative analgesia } \\
\cline { 1 - 3 } Group & Dexmedmotidine & Ketamine & \\
\cline { 1 - 3 } Variable & Mean \pm SD & Mean \pm SD & \\
\hline $\begin{array}{lccl}\text { Postoperative mean } \\
\text { pain (h) }\end{array}$ & $12.6 \pm 2.8$ & $5.1 \pm 13.3$ & 0.04 \\
\hline
\end{tabular}

duration of analgesia in the ketamine group was greater than that of dexmedmotidine.

\section{Discussion}

Cesarean section refers to the removal of the embryo from the abdominal wall and the uterus. This is done to ensure the health of the mother and the baby. ${ }^{16}$ Cesarean section is characterized by acute post-operative pain. Effective management of post-operative pain is part of the surgical process and involves a multifaceted approach in which different drugs are used with different mechanisms and prescriptions. The administration of non-opioid analgesics is an essential component of multi-faceted pain management programs. ${ }^{17}$ Therefore, the present study aimed to investigate the effect of dexmedmotidine and ketamine, using intra-peritoneal method, in order to control postoperative pain in patients who underwent cesarean section. In this study, there was no significant difference between the two groups in terms of the pain scores at different hours after surgery $(P \geq 0.05)$. Furthermore, the mean pain scores were similar in patients at different hours after surgery. In this study, there was a significant difference between the two groups in terms of drug abuse $12 \mathrm{~h}$ after the surgery $(P$ $=0.03$ ). Moreover, the mean opioid use in the ketamine group was lower than that of dexmedmotidine. Similarly, Shariat Moharari et al. investigated the effect of ketamine and bupivacaine on analgesia after laparoscopic cholecystectomy. Shariat Moharari et al. found that the duration of extubation in the ketamine group was higher. Moreover, pain was significantly lower in the ketamine group $6 \mathrm{~h}$ after surgery. The total amount of meperidine consumed within the 24 -h period was lower in the ketamine group. In addition, the drug demand decreased. There was an increase in the duration of extubation. ${ }^{18}$ In this study, there was a significant difference between the two groups (dexmedmotidine and ketamine) in terms of mean postoperative anesthesia. Moreover, the mean postoperative 
analgesia in the ketamine group was significantly higher than that of dexmedmotidine $(P=0.04)$. Oza et al. aimed at comparing the effects of intraperitoneal anesthesia of bupivacaine and dexmedmotidine only after laparoscopic surgery. Oza et al. investigated the duration of analgesia in the dexmedmotidine group. They found that the rate of intake of analgesics in the dexmedmotidine and bupivacaine was 1.76 and 2.56, respectively. This difference was significant $(P \otimes 0.05)$. The mean analgesia was lower in the 24 -h period in the dexmedmotidine group ( $P$-value of -0.05$)$. There was a significant difference the two groups in terms of pain up to $12 \mathrm{~h}$ after the surgery, and the pain was lower in the dexmedmotidine group $(P<0.05)$. Bupivacaine and dexmedmotidine increased the duration of analgesia. They also reduced the number of applications for postoperative analgesia. ${ }^{19}$ Chiruvella et al. used dexmedmotidine and rupivacaine to manage the postoperative pain after hysterectomy. The pain was lower in the rupivacaine and dexmedmotidine groups. The duration of analgesia was higher in this group. They stated that interferon dexmedotomidine can reduce pain and drug use. ${ }^{20}$ Their results are not consistent with those of our study. In our study, the mean duration of analgesia was lower in the intraperitoneal ketamine group. In our study, ketamine and dexmedmotidine were given intraperitoneally.

\section{Conclusion}

According to the results, the mean opioid use in the ketamine group was lower than that of dexmedmotidine. Moreover, the mean postoperative analgesia in the ketamine group was higher than that of dexmedmotidine. Therefore, it can be concluded that ketamine has a better effect on pain relief after cesarean section.

\section{Conflicts of Interest}

None.

\section{References}

1 Shang AB, Gan TJ. Optimising postoperative pain management in the ambulatory patient. Drugs 2003;6:855-867.

2 Agah M, Dabbagh A, Hashemi M. Evaluation of the effect of intravenous magnesium on acute postoperative pain in elective orthopedic surgeries of the lower limb. Pajoohandeh 2006:1:149-152.

3 Al-Mustafa MM, Abu-Halaweh SA, Aloweidi AS, Murshidi MM, Ammari BA Awwad ZM, et al. Effect of dexmedetomidine added to spinal bupivacaine for urological procedures. Saudi Med J. 2009:30:365-370.

4 Festin MR, Laopaiboon M, Eriksson C, Rubertsson C, Astrid Nystedt A, Hildingsson I. Cesarean section. Birt. 2011:4:12-18.

5 Eltzschig HK, Lieberman ES, Camann WR. Regional anesthesia and analgesia for labor and delivery. N Engl J Med. 2003:34:319-332.

6 Ghadamgahi HB. Prevalence and causes of cesarean in Semnan. Arch J. 2007:4:52-58.

7 Wallace DH, Leveno KJ, Cunningham FG, Giesecke AH, Shearer VE, Sidawi JE. Randomized comparison of general and regional anesthesia for cesarean delivery in pregnancies complicated by severe preeclampisia. Obsetet Gynecol. 1995;8:193-199.

8 McVey JD, Tobias JD. Dexmedetomidine and ketamine for sedation during spinal anesthesia in children. J Clin. 2010;22:538-545

9 Gramke HF, de Rijke JM, van Kleef M, Raps F, Kessels AG, Peters ML, et al. The prevalence of postoperative pain in a cross-sectional group of patients after day case surgery in a university hospital. Clin J Pain. 2007:23:543-548.

10 Madineh H, Rajaei Esfahani M, Ghaherei H, Akhlaghi M, Gangi F. The effect of intravenous low dose ketamine on postoperative pain. J Shahrekord Univ Med Sci. 2005:7:29-34

11 Khan M, Raza R, Zafar S, Shamim F, Raza SA, Pal KM, et al. Intraperitoneal lignocain versus bupivacain after laparoscopic cholecystectomy. J Surg Res. 2012;178:62-69.

12 Kahokehr A. Intraperitoneal local anesthetic for postoperative pain. Saudi J Anaesth. 2013;7:55-60.

13 Argiriadou H, Himmelseher S, Papagiannopoulou P, Georgiou M, Kanakoudis F, Giala M, et al. Improvement of pain treatment after major abdominal surgery by intravenous S+-ketamine. Anesth Analg. 2004;98:1413-1418.

14 Selim MF, Elnabtity AM, Hasan AM. Comparative evaluation of epidural bupivacaine - dexmedetomidine and bupivacaine -fentanyl on Doppler velocimetry of uterine and umbilical arteries during labor. J Prenat Med. 2012;6:47-54.

15 Kamali A, Azadfar R, Pazuki S, Shokrpour M. Comparison of dexmedetomidine and fentanyl as an adjuvant to lidocaine 5\% for spina anesthesia in women candidate for elective caesarean. Open Access Maced J Med Sci. 2018;6:1862-1867

16 Martin TC, Bell P, Ogunbiyi I. Comparison of general anesthesia and spinal anesthesia for cesarean section in Antigua and Barbuda. West Indian Med J. 2007:56:330-333.

17 Shohani M, Rasoli M, Maleki F. Comparison study of pain after cesarean section by general and spinal anesthesia. Sci J llam Univ Med Sci. 2012;21:17-25.

18 Shariat Moharari R, Hadavi M, Pourfakhr P, Najafi A, Etezadi F, Khajavi MR. Evaluation of the postoperative analgesic efficacy of intraperitoneal ketamine compared with bupivacain in laparascopic cholecystectomy. Arch Anesth Crit Care 2016;2:146-149

19 Oza VP, Parmar V, Badheka J, Nanavati DS, Taur P, Rajyaguru AM. Comparative study of postoperative analgesic effect of intraperitoneal instillation of dexmedetomidine with bupivacaine and bupivacaine alone after laparoscopic surgery. J Minim Access Surg. 2016;12:260-264

20 Chiruvella S, Nallam S. Interaperitoneal insillation of ropivacain plus dexmedetomidine for pain relief after laparoscopic hysterectomy: a comparison with ropivacaine alone. J Dr NTR Univ Health Sci. 2016;5:93-97.

This work is licensed under a Creative Commons Attribution-NonCommercial 3.0 Unported License which allows users to read, copy, distribute and make derivative works for non-commercial purposes from the material, as long as the author of the original work is cited properly. 